\title{
SANDOS-1778C \\ Systems Engineering: A Problem of Perception
}

\author{
Michael Senglaub Ph.D. \\ Sandia National Laboratories \\ PO Box 5800 MS0475 \\ Albuquerque, NM 87185 \\ 505.844.9244 \\ 505.844.8923 Fax \\ mesengl@sandia.gov
}

\begin{abstract}
The characterization of systems engineering as a discipline, process, procedure or a set of heuristics will have an impact on the implementation strategy, the training methodology, and operational environment. The systems engineering upgrade activities in the New Mexico Weapons Development Center and a search of systems engineering related information provides evidence of a degree of ambiguity in this characterization of systems engineering. A case is made in this article for systems engineering being the engineering discipline applied to the science of complexity. Implications of this characterization and some generic issues are delineated with the goal of providing an enterprise with a starting point for developing its business environment.
\end{abstract}

\section{INTRODUCTION}

There is a growing recognition in a number of Department of Energy (DOE) organizations that systems engineering may provide a foundation for solving many of the complex design and analysis problems we are going to face in the near term and in the future. The New Mexico Weapons Development Center is actively working to improve its ability to conduct activities within the Center's sphere of responsibility. At the preliminary stages in our organization's activities, a number of lessons are emerging that might prove beneficial to other organizations involved in a similar effort. Activities associated with identifying and implementing skills fundamental to systems engineering can be highly divisive. A factor contributing to this problem may be a fundamental misperception of systems engineering.

There appears to be a number of divergent opinions, implicit and explicit, concerning the nature of systems engineering. The issues concern the characterization of systems engineering as a discipline, a process, or assets of heuristics. Each characterization has implications in training, creativity, execution, and the relationship within an enterprise. In order to explore these theses and begin to develop foundations on which to build a philosophy of systems engineering, it might prove useful to examine the fundamental precept of engineering. One definition of engineering proposed by the Engineers' Council for Professional Development (ECPD) is that "Engineering is the profession in which a knowledge of the mathematical and natural sciences gained by study, experience, and practice is applied with judgement to develop ways to utilize, economically, the materials and forces of nature for the benefit of mankind." A slightly more abstract definition states that "Engineering basically involves the creation of something useful from a body of scientific knowledge by employing technical exper-

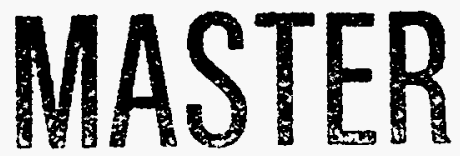


tise, individual skills in the application of transformational methods, and personnel with the proper attitudinal characteristics" [1].

The two definitions have similarities and differences that reflect the breadth of engineering activities. The definitions indicate that the product of engineering is the result of an extensive practical knowledge of science and mathematics and generates some form of benefit. It is interesting that the second definition expresses the creative aspects and individual skills of engineering. These definitions become important when considering the characterization of systems engineering.

\section{Systems}

We find in the systems engineering literature that there is ambiguity in the definition of systems as well as systems engineering. B.S. Blanchard characterizes a system as “... a nucleus of elements structured in such a manner as to accomplish a function designed to satisfy an identified need" [1]. A somewhat more abstract definition of a system is, "A system creates outputs based on inputs, over which it has no direct control" [2]. The Army Field Manual defines systems as

A composite of equipment, skills and techniques capable of performing and/or supporting an operational role. A complete system includes related facilities, equipment, material, services, software, technical data and personnel required for its operation and support to the degree that it can be considered a self-sufficient unit in its intended operation and/or support environment. The system is employed operationally and supported logistically [3].

The most interesting concept of a system was suggested by Dr. E. W. Aslaksen that the notion of a "system" in its purest form is an abstract concept. By this he is implying that like the number "7," which is a description of quantity, system is a description of complexity. The intent in this case is to provide a foundation from which to develop a set of operators, like mathematical operators, which could be used to operate on a "system" to provide useful design/analysis information. In his paradigm Dr. Aslaksen suggests the following system definition:

A system consists of three related sets: A set of functional elements; a set of internal interactions; and a set of external interactions. A functional element is a description of an entity, which may be physical (e.g. hardware, software, workpackage, person) or formal (e.g. position, concept), but such that the element is defined only by its interactions with the surroundings of the entity [4].

\section{Systems Engineering}

The difficulty of defining a system is compounded when attempts are made to define systems engineering. As indicated, the spectrum of interpretations of systems engineering ranges from an engineering discipline to a process. S. A. Hyer characterizes systems engineering as "... a series of activities in which one applies principles and technology from multiple disciplines in a team environment to define and solve problems" [5]. A similar process-oriented definition was proposed which states that "Systems engineering per se is not considered as an engineering discipline in the same context as the technical speciality areas..." [1]. Some of the speciality disciplines he was referring to included reliability, logistics, human factors, and maintainability engineering. He goes on the state that "... systems engineering is a process employed in the evolution of system 
development (particularly for large systems) from the point when a need is identified through production and/or construction and ultimate deployment of that system in the field for consumer use" [1].

Alternate views of systems engineering are proposed by N. B. Reilly, in which he recognizes and discusses the dynamic nature of systems engineering. He provides a historical glimpse of the evolutionary aspects of the field from a staircase model to early prototype to spiral, and of late, the rapid development models. Within this evolutionary context Reilly states:

Early prototypes penetrates to the requirements level. The spiral model penetrates to the design level. Rapid development, the most recent innovation, engulfs the entire process, resulting in repeated operational deliveries. Each paradigm continues to have its useful niche. Awareness and proper application of the evolutionary perspective can be of significant value to project and systems engineers. Selecting the wrong model for a given task will increase the probability of turmoil and, as history warns us, even failure [6].

The truly innovative and insightful view of systems engineering comes from Dr. Aslaksen when he states that "... systems engineering is to classical engineering what statistical mechanics is to mechanics; a fact that was realized long ago but has since been somewhat forgotten" [7]. He feels that much of the work in systems engineering intended to develop techniques and tools for use in systems design is missing: "... the inherently probabilistic or statistical nature of systems engineering and its consequent ability to handle "fuzzy" problems and risk" [4]. I think that this aspect is repeatedly exhibited in our continued treatment of system design as a deterministic problem rather than accept the stochastic nature of the problem and employ techniques that are better suited for this class of problems.

\section{Definitions and Examples}

The extensive prelude is intended to provide a setting from which to identify implications of the varying concepts in systems engineering. The next sections provide definitions and representative examples of processes, procedures and disciplines. This information will be used as the basis for the assertion that systems engineering is a discipline.

Processes and Procedures. Webster defines process as "...the action of continuously going along through each of a succession of acts, events, or developmental stages." Procedure is defined as "...a series of steps followed in a regular orderly defined way." The difference in these definitions is minimal and possibly subtle if you are not looking for the differences. The principle difference is that a procedure executes steps in a specific order.

Installation of a software package on a PC or workstation is an example of a procedure. We find that much of the PC software comes with an installation script which executes a series of functions required to install the product. In the unix environment software installation is documented in a manual that is included with the product. Part of the reason for this is that product installation varies based on the workstation configuration. The unix installation, in this case, may be more appropriately characterized as a process. Activities are defined that have a common start point and delineate the expected endpoint

Admittedly the example is rather simplistic; however, it does provide some interesting 
characteristics. The first characteristic of processes and procedures is that the requirements associated with training are very simple. The knowledge to perform a set of activities can be acquired by memorization or by simply maintaining an accessible copy of the processor or procedure. This is not to imply that the underlying skills, i.e. computer administration are simple and can be acquired in an unsophisticated manner. A second characteristic of a procedure or a process is that the adaptability to modified situations is severely limited. As operating systems change, or hardware is upgraded, the process for installing a software product must be redefined in order for it to be effective or even functional.

Process vs. Procedure. We might consider a dimensional analogy to better understand the concepts of processes and procedures by solving a problem of traveling from city " $A$ " to city "B," with a number of potential refueling points for use during the journey. A process description would indicate that you start at "A," monitor your fuel, refuel when the tank gets low, and terminate the journey once you have reached "B." A procedure on the other hand, defines the start point "A," the first refueling stop, each succeeding refueling point, and termination at location "B." Examination of these two activities indicates that the difference between the two activities is the addition of a "time-like dimension" to the procedure. I contend that the seqencing of events, i.e. a procedure, adds a pseudo dimension of time to a process.

Discipline. According to Webster's Third New International Dictionary, discipline is "...a subject that is taught: a branch of learning: a field of study," "... the training or experience that corrects, molds, strengthens or perfects esp. the mental faculties or moral character in order to acquire some coveted knowledge or skill."
Quantum physics is an example of a relatively sophisticated discipline. The quantum physicist develops the skills required to solve Schroedinger's wave equation, which is a correlation that describes the dynamic behavior of a particle in a force field that can be represented by a potential energy. Skills required to work in this field include advanced mathematics, including group theory, computer simulation, test, and an ability to easily move between the abstract and physical worlds. A student of quantum physics is initially presented with the problem of solving Schroedinger's equation using a "square well" potential. In solving this problem the student is provided with guidelines and "heuristics" to solve this simplest of all problems in quantum mechanics. Treating more realistic or complex problems requires greater sophistication. The WKB Method [8] is an approach for solving complex quantum problems that makes approximations to Schroedinger's equation. The WKB solution process provides guidance and limits of applicability for finding solutions. As the complexity of problems increases, more sophisticated mathematical and computer simulation techniques are employed to develop solutions. The training process objective is to develop skills that enable the quantum physicist to begin solving new physical problems for which known solutions do not exist and for which the "square well potential" and WKB process are inadequate.

Karate is an interesting example of a discipline that clearly demonstrates the utilization of processes and procedures to develop the skills associated with the discipline. The discipline of karate is intended to provide the student with the ability to formulate solutions in a combat environment. This characterization of combat, being an intellectual as well as physical activity, applies to strategic engagements of historical scale to a "street 
fight." In karate, "forms" are developed by the masters of the discipline and are examples of a tactical application of a fundamental skill set. Each form represents a different tactical scenario. The forms practiced by the student can be characterized as procedures, which were developed by a master through a training process to help the students improve their skills. The process aspects of these forms are exemplified by the instructor's encouragement to analyze the form, to examine tactical alternatives that are consistent with the situation considered in the form.

There are fundamental common characteristics of these highly divergent disciplines. The training required in order to achieve a level of proficiency can be extensive. It is also evident that there is always opportunity for improving the skills and application of these skills in the discipline. As a practicing quantum physicist, research and study represent the endeavor to improve one's ability to solve new problems.

Examination of these disciplines in greater depth shows that as proficiency in a discipline increases, the ability to apply the inherent skills in new situations becomes easier. If quantum physics were a process, the quantum physicist might try to solve a manybody nuclear problem with a "square well potential." This approach would likely lead to devastating failure. Likewise, students of Karate would suffer severe repercussions if they were to try a counter an overhand knife strike with a classic down block. The adaptability of a discipline becomes automatic as proficiency in the skills comprising a discipline improve. Students of Karate recognize that the ability to execute a form does not (1) imply that they have mastered a skill or a set. of skills, or (2) that they have become an expert in the martial arts.

\section{SYSTEMS ENGINEERING CHARAC- TERIZATION}

Part of the problem in characterizing systems engineering is determining when the application of systems engineering skills transition from optional to mandatory. We know that for problems of limited system scope it may be possible to employ skills from a single correlated discipline to solve the problem. Under those conditions it may be possible, with the aid of a set of heuristics or a process description, for a relatively inexperienced engineer to arrive at a solution. As the system scope increases in complexity, new considerations and skills need to be incorporated into the problem solving activities. Each discipline, possessing a unique view of the problem and unique solution methodologies, would arrive at different and possibly conflicting solutions to the problem. At some point the plethora of possible solution methodologies would drag the problem solving activities to a halt because of the sheer number of solution approaches that would have to be considered. It is under these conditions that the individual who understands the relative importance of the different skill sets and the advantages and disadvantages of different solution methodologies can add value and move a problem solving activity in the direction of an acceptable solution.

The supposition that systems engineering is a discipline as opposed to a process requires a closer examination of the relationships between processes, procedures, heuristics and disciplines. If we examine the role systems engineering plays in problem solving and the skill sets required in order to properly execute a systems engineering activity, we begin to see the parallels between the engineering and science disciplines and systems engineering. If, as systems engineers, we deal with a very narrowly focused set of problems or problems of marginal complex- 
ity, we could operate with a process characterization of systems engineering. However, if we are to work in an environment of evolving projects, new technologies, and increasing system complexities, we need to recognize that a process interpretation will not suffice, and eventually we will generate very poor solutions to the problems being addressed. The second part of Webster's definition of a discipline and the examples of disciplines discussed lead to the assertion that systems engineering is a discipline.

It can be argued that heuristics represent a block of information that provides guidance for performing a set of activities, for example, solving a specific thermodynamics problem. These heuristics are based on the application of a suite of disciplines to solve a problem or perform an activity. They represent an encapsulation and distillation of the knowledge of the suite of disciplines. A process also represents the product of the application of a suite of disciplines. Figure 1 attempts to demonstrate the levels of abstraction associated with the definitions and characterizations discussed in the proceeding sections.

The figure identifies relationships between a discipline, process and procedure. The contention is that procedures are developed based on a process which identifies solution approaches for classes of problems, and that a process is developed based on knowledge associated with a discipline or set of disciplines.

We saw that the development of the WKB method in quantum physics was a process for solving a specific class of quantum physics problems. We can also see that the forms associated with karate represent the result of the discipline of the martial artist being applied to solve a training problem. What is being argued is that even if there is disagreement about wether systems engineering is a process or a discipline, the fact that a process or a set of heuristics is founded in the application of a set of disciplines implies that systems engineering must be considered a discipline.

\section{Systems Engineering Interpretation}

There also appears to be differences in the interpretation of the responsibilities associated with a systems engineering implementation in an enterprise. There appears to be a management facet or interpretation as well as the problem solving interpretation of systems engineering. Hyer's paper [7] may be the most cogent example of the different interpretations of systems engineering. The figure Hyer included in his paper, which is approximated below, demonstrates pictorially the qualitative relationships between the "depth" and "breadth" of knowledge and a

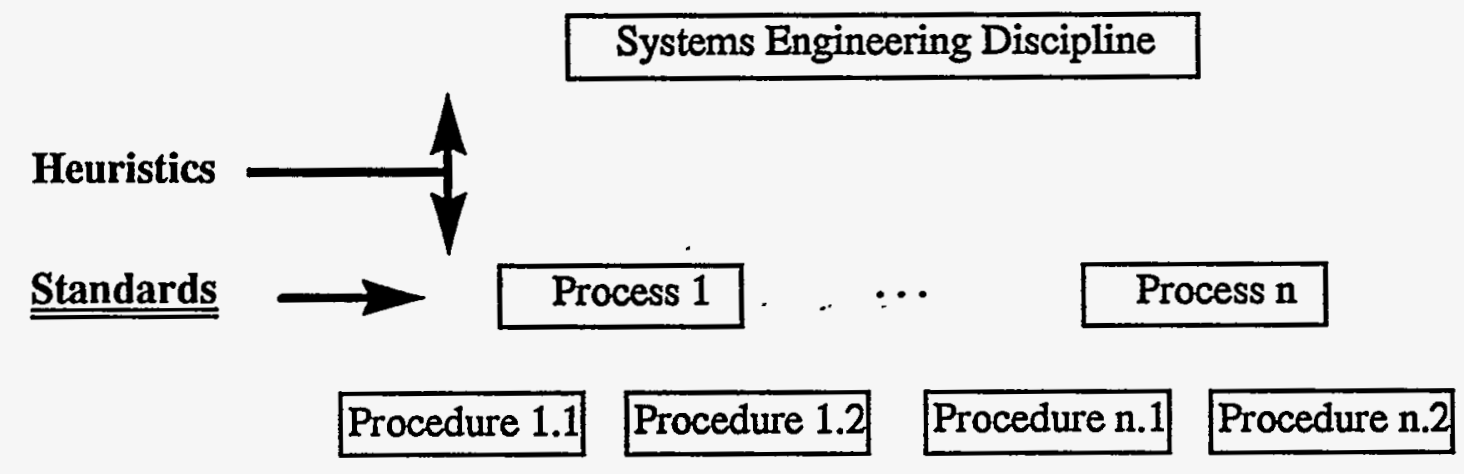

Figure 1. Heiarchical relationship of disciplines, processes, procedures and heuristics. 
characterization of an engineering professional. The interesting aspect is the region covered by systems engineering, which includes a significant depth as well as breadth of knowledge.

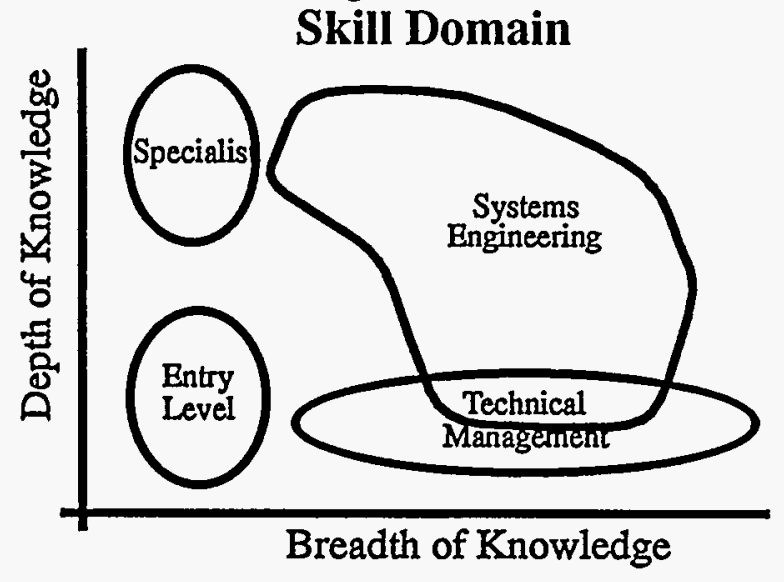

Figure 2. Approximation of Hyer's skills chart.

The second region of importance in Figure 2 is the technical management skill sets. This covers comparable breadth but does not approach the depth that is characteristic of systems engineering. It appears that in some interpretations of systems engineering there is the belief that technical management and systems engineering are synonymous. For a systems engineer, there may be technical management responsibilities, but there is a much broader interpretation that must be considered. It is this broader interpretation that additionally supports the contention that systems engineering is a discipline.

\section{SO WHAT?}

It is important to recognize that as technology evolves we are faced with a more complex set of problems requiring solution. Solutions to these problems are increasingly being defined through a hybrid of technologies. We have traditionally tried to find the "magic bullet" technology to solve new more complex problems; often a better approach would be to consider hybridized solutions to these problems. This solution paradigm is one facet of the "complexity" issues associated with the systems engineering discipline. In dealing with complexity and complex design problems, we need to be able to examine and apply the latest analytical technologies to solving these multi-disciplinary problems. We need to be able to apply biologically based algorithms, chaos theory, game theory, statistical theory, and others to the problems we are encountering today and to the evermore complex problems of the future.

With the recognition that systems engineering is a discipline goes the implicit recognition that being able to "exercise" a process description does not produce a systems engineer. A second issue associated with this recognition is that, like a mechanical engineer or an electrical engineer, there exist an environment and suites of tools that support and enhance the problem solving capabilities of the systems engineer. Finally, the training of systems engineers comes from an experienced application of the fundamental skills associated with the discipline. Single or narrowly defined applications of the discipline do not enhance the capabilities of systems engineers in the application of their skills for solving new problems. If the quantum physicists solve only "square well potential" problems, they are ill suited to solve more complex quantum problems.

\section{The Starting Point}

The foundation from which an organization needs to initiate its upgrade or implementation activities is the recognition that, like other engineering specialities, systems engineering is a problem-solving discipline. The to develop a formal systems engineering capability in an enterprise is not sufficient to realize the capability. A number of issues must be formally addressed if an organiza- 
tion is to develop/enhance its systems engineering capabilities. The enterprise must (1) permit the trained systems engineer to apply their systems engineering skills, (2) implement and utilize a detailed "lessons learned" structure, (3) provide/develop an intense broad based training program, (4) provide support for the development of the systems engineering operational environment, and (5) develop or allow for the development of a business environment that optimizes the implementation of systems engineering.

Skills Employment Issue. The first issue involves the organizational requirement that the systems engineers be allowed to apply their skills. A well-planned systems engineering activity involves a great deal of preliminary planning and analysis. In today's environment of severely constrained development schedules, there is a tendency to initiate synthesis before sufficient information is generated and planning is complete. It is a natural tendency but it can be counter-productive in a systems engineering enterprise. Along the same lines it is important that systems engineering activities be initiated prior to the allocation of engineering resources to a project prior to a need. This can be highly cost ineffective, adding non-value added cost at the start of a project and requiring a greater amount of effort to redirect efforts when it is determined that the design direction may be in error.

Lessons Learned Issue. The second issue involves implementation of a lessons learned process in conjunction with systems engineering activities. The systems engineer learns through experience. With fewer initiated projects in an organization, it becomes extremely important for a greater number of engineers to benefit from the experiences gained by a project lead. Documentation of approaches for solving a problem including approaches that did and did not succeed, as well as the rationale for the approaches, provides other engineers in an organization with a vicarious experience of the application of the systems engineering discipline. An alternative approach that can be used in conjunction with the lessons learned documentation is that lead engineers be tasked with producing a short course that conveys in an encapsulated manner the experience they have gained at the conclusion of the activity. This approach may also leverage the organization's experience through the development and examination of different solution approaches identified through the course work.

Training Issue. The training program that needs to be implemented should be rather intense. Recognition that systems engineering is a discipline requires a different approach to education and training. It must be recognized that as technologies change and new approaches to solving problems emerge they need to be added to the tool suite of the systems engineer. This would imply that significant effort needs to be continually expended by a systems engineer in training or through and an organizational research activity. Initial impatience with systems engineering short courses has been tempered with the realization that the multiple facets associated with systems engineering do not invalidate any training related to systems engineering. This leads to a postulation that the training program needs to reflect the various facets and is properly achieved through a multi-faceted training program, i.e. contractors, short courses, in-house training, and classes developed by the practicing engineers of an organization.

As indicated earlier, a narrow application of systems engineering skills does not provide engineers with the experiences they need to develop as systems engineers. The enterprise needs to identify and actively seek 
opportunities for applying systems engineering to problems that might not be typically considered their "principle line of business." To broaden an organization's experience it might also prove useful for the project lead to "contract" another engineer in the same organization to perform a specific systems engineering function. The approach could leverage an organization's experience in systems engineering by expanding the number of people involved without adding overhead to a project. This approach also provides a natural mentoring structure, selecting a new engineer for the contracted activity, and using the lead or an organization expert as the engineers mentor on the activity to provide guidance and advise.

Systems Engineering Environment Issue. The fourth issue to be directly addressed is the issue of operational environment. Issues of tools, networks, platforms, and resource support must be resolved prior to an engineer being assigned a systems engineering activity. It is not cost effective for the systems engineer to lead a project and work to identify and bring on line new tools and techniques associated with systems engineering. As part of a project, the mechanical engineer is not typically tasked with performing a structural analysis as well as developing the tools and the platforms needed to complete the activities. The infra-structure needed by the systems engineer should be in place prior to assignment to a project. Failing to perform these activities is similar to requiring a blacksmith to operate without furnaces, welding equipment, or even hammers.

Enterprise Issue. The final issue to be considered is somewhat more abstract than the preceding issues. The issue that needs to be considered is how the systems engineering enterprise needs to be established and how it will conduct business within an organization. Inappropriate implementation of the sys- tems engineering enterprise can potentially mitigate the effectiveness of that organization. The tools and skills of the systems engineer can and should be applied to identify the optimal business processes for the systems engineering enterprise. Since each enterprise must operate in a unique business environment, the optimal enterprise will differ from organization to organization. This design approach to developing the enterprise can help to identify costly or nonvalue added activities that should be pruned from the business practices.

\section{SUMMARY}

Systems engineering is the discipline in which engineering disciplines are applied to the science of complexity. With this recognition comes a set of fundamental requirements relating to training and implementation in an organization. We need to recognize that the processes and standards associated with systems engineering are exemplars of the application of the discipline to a class of problems. There needs to be a recognition that processes and procedures represent single instances, from a continuous space of solution approaches, of the application of the systems engineering problemsolving discipline.

\section{REFERENCES}

[1] B.S. Blanchard; "Engineering Organization and Management"; Prentice-Hall Inc., Englewood Cliffs, NJ; 1976; ISBN 0-13279430-6.

[2] W. Chapman, A.T. Bahill, A.W. Wymore; "Engineering modeling and Design"; CRC Press, Boca Raton Fl 1992; ISBN 08493-8011-1. 
[3] United States Army Field manual - Systems Engineering, FM 77078

[4] E.W. Aslaksen; "A Leadership Role for NCOSE"; Proceedings of the Forth International Symposium of the National Council On Systems Engineering(NCOSE); August, 1994; pp.225-229.

[5] S.A. Hyer, "An Integrated Approach to Systems Engineering Training"; Proceedings of the Forth International Symposium of the National Council On Systems Engineering(NCOSE); August, 1994; pp.347-354.

[6] N. B. Reilly; "Successful Systems Engineering"; Van Notrand Reinhold, 1993; New York, NY, ISBN 0-442-01586-0

[7] von Neumann, The General an Logical Theory of Automata, paper read at Hixon Symposium, 20 September 1948, Pasadena CA; Collected Works of John von Neumann, A.H. Taub, Ed., Pergammon Press, Oxford(1961-63), vol. 5 pp. 288-328.

[8] A. Messiah; "Quantum Mechanics, Volume I"; John Wiley \& Sons, Inc., New York NY, 1966

\section{AUTHOR BIOGRAPHY}

Michael E. Senglaub has worked in a number of engineering departments at Sandia National Laboratories since 1979. Prior to that time he has worked as a research engineer at Argonne National Laboratories and the $\mathrm{KfK}$ in Karlsruhe Germany. He has a $\mathrm{Ph} . \mathrm{D}$. in Engineering Science from Northwestern University, a M.S. in Mechanical/ Nuclear Engineering from the University of Texas, Austin and a B.S. in Physics from the University of Texas, Austin. He is a member of MORS and NCOSE.

This work was supported by the United States Department of Energy under Contract DE-ACO494AL85000.

\section{DISCLAIMER}

\begin{abstract}
This report was prepared as an account of work sponsored by an agency of the United States Government. Neither the United States Government nor any agency thereof, nor any of their employees, makes any warranty, express or implied, or assumes any legal liability or responsibility for the accuracy, completeness, or usefulness of any information, apparatus, product, or process disclosed, or represents that its use would not infringe privately owned rights. Reference herein to any specific commercial product, process, or service by trade name, trademark, manufacturer, or otherwise does not necessarily constitute or imply its endorsement, recommendation, or favoring by the United States Government or any agency thereof. The views and opinions of authors expressed herein do not necessarily state or reflect those of the United States Government or any agency thereof.
\end{abstract}

